\title{
Non-equilibrium phenomena in the QCD phase transition
}

\author{
E. N. Saridakis * N. G. Antoniou $₫$ F. K. Diakonos $\sharp$ and N. Tetradis \\ Physics Department, University of Athens, 15771 Athens, Greece
}

\begin{abstract}
Within the context of the linear $\sigma$-model for two flavours, we investigate non-equilibrium phenomena that may occur during the QCD chiral phase transition in heavy-ion collisions. We assume that the chiral symmetry breaking is followed by a rapid quench so that the system falls out of thermal equilibrium. We study the mechanism for the amplification of the pion field during the oscillations of the $\sigma$-field towards and around its new minimum. We show that the pion spectrum develops a characteristic pronounced peak at low momenta.
\end{abstract}

\section{THE MODEL}

Experiments at RHIC and LHC are expected to probe many questions in strong interaction physics. One major area of interest concerns the chiral phase transition. For given baryon-number chemical potential $\mu$ there exists a critical temperature $T_{c r}$ above which the system lies in the chirally symmetric state. As the temperature decreases below $T_{c r}$ the system moves into the chirally broken phase. It is believed that, for two flavours and zero quark masses, there is a 1st-order phase transition line on the $(T, \mu)$ surface at large $\mu$ [1]. This line ends at a tri-critical point beyond which the phase transitions become 2 nd order. The line of 2 nd-order transitions ends on the $\mu=0$ axis. In the case of non-zero quark masses, the 1st-order line ends at a critical point, beyond which the 2nd-order transitions are replaced by analytical crossovers.

This phase diagram has been discussed within various frameworks. Our interest lies in the study of possible non-equilibrium phenomena that may occur during the phase transition. In particular we would like to study the possibility that the system falls out of thermal equilibrium through rapid expansion. This is a realistic possibility in the framework of heavy-ion collisions.

The scenario we have in mind assumes an initial thermalization at a sufficiently high temperature for the system to move into the chirally symmetric phase. The subsequent fast expansion generates deviations from thermal equilibrium. We model this process by a quench during which the volume of the system increases instantaneously by a certain factor, with the number densities of the various particles decreasing by the same factor.

We consider only the two lightest flavours and neglect the effects of the strange quark. As an effective description of the chiral theory we use the $\sigma$-model [2, 3]. The Lagrangian density is

$$
\mathcal{L}=\frac{1}{2}\left(\partial_{\mu} \sigma \partial^{\mu} \sigma+\partial_{\mu} \vec{\pi} \partial^{\mu} \vec{\pi}\right)-V(\sigma, \vec{\pi})
$$

with the potential

$$
V(\sigma, \vec{\pi})=\frac{\lambda^{2}}{4}\left(\sigma^{2}+\vec{\pi}^{2}-v^{2}\right)^{2}+\frac{m_{\pi}^{2}}{2}\left(\sigma^{2}+\vec{\pi}^{2}-2 v \sigma+v^{2}\right) .
$$

The last term in the potential accounts for the explicit chiral symmetry breaking by the quark masses. The scalar field $\sigma$ together with the pseudoscalar field $\vec{\pi}=\left(\pi^{+}, \pi_{0}, \pi^{-}\right)$form a chiral field $\Phi=(\sigma, \vec{\pi})$. When the symmetry is restored at high temperatures in the absence of the second term in the potential, the system lies in the symmetric state $\langle\sigma\rangle=\langle\vec{\pi}\rangle=0$. However, in the presence of the explicit symmetry breaking term in (2), the expectation value of the $\sigma$-field never vanishes, so that the chiral symmetry is never completely restored. At zero temperature and chemical potential the expectation values of the fields become $\langle\sigma\rangle=v=f_{\pi}$ and $\langle\vec{\pi}\rangle=0$, where $f_{\pi}$ is the pion decay constant. We fix the parameters of the Lagrangian using the phenomenological values $m_{\pi} \approx 139 \mathrm{MeV}$, $m_{\sigma}=\sqrt{2 \lambda^{2} f_{\pi}^{2}+m_{\pi}^{2}} \approx 600 \mathrm{MeV}, v \approx 87.4 \mathrm{MeV}$, which yield $\lambda^{2} \simeq 20$.

\footnotetext{
*Electronic address: msaridak@phys.uoa.gr

† Electronic address: nantonio@cc.uoa.gr

$\ddagger$ Electronic address: fdiakono@cc.uoa.gr

$\S$ Electronic address: ntetrad@cc.uoa.gr
} 


\section{EQUATIONS OF MOTION}

The equations of motion resulting from (1) are:

$$
\begin{aligned}
& \partial_{\mu} \partial^{\mu} \sigma+\lambda^{2}\left(\sigma^{2}+\vec{\pi}^{2}-v^{2}\right) \sigma+m_{\pi}^{2} \sigma=v m_{\pi}^{2} \\
& \partial_{\mu} \partial^{\mu} \vec{\pi}+\lambda^{2}\left(\sigma^{2}+\vec{\pi}^{2}-v^{2}\right) \vec{\pi}+m_{\pi}^{2} \vec{\pi}=0 .
\end{aligned}
$$

We neglect the fluctuations of $\sigma$, while we treat $\vec{\pi}(\vec{x}, t)$ as a quantum field:

$$
\vec{\pi}(\vec{x}, t)=\int \frac{d^{3} k}{(2 \pi)^{3}}\left(a_{k, v} \vec{f}_{k}(t) e^{-i \vec{k} \vec{x}}+a_{k, v}^{\dagger} \vec{f}_{k}^{\star}(t) e^{i \vec{k} \vec{x}}\right) .
$$

The creation and annihilation operators $a_{k, v}^{\dagger}, a_{k, v}$ are defined in the interaction picture at the vacuum corresponding to the minimum $v$ of the potential, and $\vec{f}_{k}(t)$ are the mode functions of the pion field.

We work within the framework of the Hartree approximation. This leads to the effective replacement [4]

- $\vec{\pi}^{2}(\vec{x}, t) \rightarrow\left\langle\vec{\pi}^{2}(\vec{x}, t)\right\rangle=\left\langle\vec{\pi}^{2}(t)\right\rangle$

- $\vec{\pi}^{2}(\vec{x}, t) \vec{\pi}(\vec{x}, t) \rightarrow \frac{5}{3}\left\langle\vec{\pi}^{2}(t)\right\rangle \vec{\pi}(\vec{x}, t)$,

with $\left\langle\pi_{i}^{2}(t)\right\rangle=\left\langle\vec{\pi}^{2}(t)\right\rangle / 3$ for $\mathrm{i}=1,2,3$. The second approximation results from the replacement of the term $\pi_{i}^{2} \pi_{j}$ by $3\left\langle\pi_{i}^{2}\right\rangle \pi_{j}$ for $j=i$, and by $\left\langle\pi_{i}^{2}\right\rangle \pi_{j}$ for $j \neq i$. Substituting the above approximations into (3) we get:

$$
\begin{gathered}
\ddot{\sigma}(t)+\lambda^{2}\left(\sigma^{2}(t)+\left\langle\vec{\pi}^{2}(t)\right\rangle-v^{2}\right) \sigma(t)+m_{\pi}^{2} \sigma(t)=v m_{\pi}^{2} \\
\overrightarrow{\tilde{f}}_{k}(t)+\left[k^{2}-\lambda^{2} v^{2}+\lambda^{2} \sigma^{2}(t)+\frac{5}{3} \lambda^{2}\left\langle\vec{\pi}^{2}(t)\right\rangle+m_{\pi}^{2}\right] \vec{f}_{k}(t)=0 .
\end{gathered}
$$

In (5), (6) $\left\langle\vec{\pi}^{2}(t)\right\rangle$ is given by

$$
\left\langle\vec{\pi}^{2}(t)\right\rangle=\int \frac{d^{3} k}{(2 \pi)^{3}} \vec{f}_{k}^{\star}(t) \vec{f}_{k}(t) .
$$

The particle density per momentum mode, for each component $i$ of the pion field is [5, 6]:

$$
n_{k i}=\frac{\omega_{k}}{2}\left(\left|f_{k i}(t)\right|^{2}+\frac{\left|\dot{f}_{k i}(t)\right|^{2}}{\omega_{k}^{2}}\right)-\frac{1}{2}
$$

with $\omega_{k}=\sqrt{k^{2}+m_{\pi}^{2}}$. For the total number of pions of all species $\left(\pi^{+}, \pi^{0}, \pi^{-}\right)$we have:

$$
\frac{N_{t o t}(t)}{V}=\sum_{i}^{3} \int \frac{d^{3} k}{(2 \pi)^{3}} n_{k i}(t)
$$

where $V$ is the volume of our system, that is the volume of the fireball in a heavy-ion collision experiment.

\section{INITIAL CONDITIONS}

Our choice of the vacuum at $v$ as our reference state has the advantage that the particle interpretation of the field $\vec{\pi}(\vec{x}, t)$ is close to the experimentally observable quantities. It requires, however, some care with respect to our choice of initial conditions for the evolution described by equations (5), (6). We assume that the fireball created by the collision is initially in local thermodynamic equilibrium, or it has been separated in Disoriented Chiral Condensates (DCCs), each one in its own local thermodynamic equilibrium. If the second case is realised, our treatment applies to the interior of one of these DCCs. The initial expectation value $\sigma_{1}$ that we consider for the $\sigma$-field is small, but non-zero, because of the explicit chiral symmetry breaking. For our calculation we use $\sigma_{1}=0.1 v$ at $T=140 \mathrm{MeV}$. 
For the pions we expect initially a thermalised gas that follows a Bose-Einstein distribution with

$$
n_{k i}^{\mathrm{eq}}=\frac{1}{e^{\frac{\omega_{k}}{T}}-1} \text {. }
$$

We assume the dispersion relation around the vacuum at $\sigma=v: \omega_{k}^{2}=k^{2}+m_{\pi}^{2}$, even though the pion mass depends on the temperature. The justification for this approximation is provided by the explicit study in [7] of the effective pion mass during the process of chiral restoration. There, it is shown that $m_{\pi}$ stays approximately constant from $T=0$ up to $T \sim 100 \mathrm{MeV}$.

The mode functions $f_{k i}(t)$, for a configuration corresponding to a non-interacting pion gas in thermal equilibrium, can be taken

$$
f_{k i}^{\mathrm{eq}}=\sqrt{n_{k i}^{\mathrm{eq}}+1 / 2} \frac{e^{-i \omega_{k} t}}{\sqrt{\omega_{k}}}
$$

in agreement with (8). In the following we restrict our analysis to large occupation numbers, and neglect the factor $1 / 2$ related to the zero point energy.

In our simplified scenario we assume an instantaneous expansion of the fireball by a volume factor $\Lambda$ (a quench). This means that the number densities of the pion gas must be reduced by the same factor. In addition, in order to be consistent with the conservation of energy, the initial value of the $\sigma$-field has to change according to the relation

$$
V\left(\sigma_{\Lambda}\right)=\frac{V\left(\sigma_{1}\right)}{\Lambda}
$$

where $\sigma_{1}$ is the value before the quench, and $\sigma_{\Lambda}$ the one after. We point out that this assumption is rather crude as it neglects possible fluctuations of $\sigma$. However, it satisfies the minimal requirement of energy conservation.

The above discussion implies that the physically motivated initial conditions for the evolution of the fields are

$$
f_{k i}(0)=\sqrt{\frac{n_{k i}^{\mathrm{eq}}}{\Lambda}} \frac{1}{\sqrt{\omega_{k}}}, \quad \dot{f}_{k i}(0)=\sqrt{\frac{n_{k i}^{\mathrm{eq}}}{\Lambda}}\left(-i \sqrt{\omega_{k}}\right)
$$

and

$$
\sigma(0)=\sigma_{\Lambda}, \quad \dot{\sigma}(0)=0
$$

These initial conditions differ from the ones assumed for particle production through inflaton decay in cosmology [8], and in some works on QCD [9, 10], as in those cases the initial particle number is taken to be zero.

\section{NON-EQUILIBRIUM EVOLUTION}

Equations (5) and (6), determining the evolution of the $\sigma$ and the pion field, constitute a non-linear integrodifferential system that is not solvable analytically. The main difficulty is related to the presence of the term $\left\langle\vec{\pi}^{2}(t)\right\rangle$ that mixes all the pion mode functions. If we ignore this term, it is possible to decouple the evolution of $\sigma$ from that of the pion field. We introduce the variable $\eta(t)=\sigma(t) / v$, and absorb $\lambda^{2} v^{2}$ in a new rescaled time variable $\tau=\lambda v t$. We also introduce the small parameter $\varepsilon=m_{\pi}^{2} /\left(2 \lambda^{2} v^{2}\right) \ll 1$. In this way we obtain

$$
\eta^{\prime \prime}(\tau)+\eta^{3}(\tau)+(2 \varepsilon-1) \eta(\tau)-2 \varepsilon=0,
$$

where primes denote differentiation with respect to $\tau$.

In zeroth order in $\varepsilon$, i.e $\varepsilon=0$, this equation can be solved in terms of elliptic functions, with the result

$$
\eta(\tau)=\frac{\eta(0)}{\operatorname{dn}\left(\tau \sqrt{1-\frac{n^{2}(0)}{2}}, q\right)}
$$

where

$$
q=\sqrt{\frac{1-\eta^{2}(0)}{1-\frac{\eta^{2}(0)}{2}}}
$$


Here dn stands for the known Jacobi elliptic function and $q$ is its modulus. (We do not use the standard symbol $k$ in order to avoid confusion with momentum.) We point out that the solution (16) holds only for the range $0 \leq \eta(0) \leq 1$, relevant to our case. For $\eta(0)>1$ the solution is expressed in terms of different elliptic functions.

Inserting the solution (16) into the equation for the pion mode functions, we obtain

$$
f_{k i}^{\prime \prime}(\tau)+\left[\frac{k^{2}}{\lambda^{2} v^{2}}+\frac{\eta^{2}(0)}{\operatorname{dn}^{2}\left(\tau \sqrt{1-\frac{\eta^{2}(0)}{2}}, q\right)}+2 \varepsilon-1\right] f_{k i}(\tau)=0 .
$$

This is the Lamé equation, for particular values of the coefficients which make it solvable in terms of Jacobi functions [11, 12].

We first investigate the zeroth order solution $(\varepsilon=0)$. As we show in the Appendix A, the solution $f_{k i}(\tau)$ of $(18)$ is a quasi-periodic function that can grow with time. Exponential amplification is obtained if the pion momentum lies in one of the zones: a) $-\infty \leq k^{2} /\left(\lambda^{2} v^{2}\right) \leq 0$, b) $\eta^{2}(0) / 2 \leq k^{2} /\left(\lambda^{2} v^{2}\right) \leq 1-\eta^{2}(0) / 2$. In our case the first zone is unphysical. Substituting $\sigma(t)=v \eta(t)$, we obtain for the remaining amplification zone

$$
\frac{\lambda}{\sqrt{2}} \sigma(0) \leq k \leq \frac{\lambda}{\sqrt{2}} \sqrt{2 v^{2}-\sigma^{2}(0)},
$$

a result valid in zeroth order in $\varepsilon=m_{\pi}^{2} /\left(2 \lambda^{2} v^{2}\right)$.

Obtaining a full analytical solution valid in first order in $\varepsilon$ is very complicated. However, it is trivial to take into account the term $2 \varepsilon$ in equation (18). Its consideration simply leads to the replacement of $k^{2} /\left(\lambda^{2} v^{2}\right)$ by $2 \varepsilon+k^{2} /\left(\lambda^{2} v^{2}\right)$. The amplification zone becomes

$$
\sqrt{\frac{\lambda^{2}}{2} \sigma^{2}(0)-m_{\pi}^{2}} \leq k \leq \sqrt{\frac{\lambda^{2}}{2}\left(2 v^{2}-\sigma^{2}(0)\right)-m_{\pi}^{2}}
$$

We emphasize that this result is not the complete answer in first order in $\varepsilon$, as equation (16) is not an exact solution of (15) for $\varepsilon \neq 0$. However, the amplification zone given by equation (20) agrees very well (within $4 \%$ ) with the amplification observed through the numerical solution of the equations.

If $\sigma(0)$ is sufficiently small $\left(\sigma(0)<\sqrt{\frac{2}{\lambda^{2}}} m_{\pi} \simeq 0.5 v\right)$ the lower end of the amplification zone (20) is at $k=0$. For larger $\sigma(0)$ the zone becomes narrower. In the extreme case $\sigma(0) \simeq v$ (when the $\sigma$-field starts its evolution very close to its minimum), the zone shrinks to a point

$$
k^{2} \simeq \frac{m_{\sigma}^{2}}{4}-m_{\pi}^{2}
$$

This is the pion momentum in the decay $\sigma \rightarrow 2 \pi$.

The form of equation (6) for the pionic modes indicates the presence of two regimes in their evolution:

a) Shortly after the quench the various modes have an effective mass term

$$
m_{e f f}^{2}=k^{2}-\lambda^{2} v^{2}+\lambda^{2} \sigma^{2}(0)+\frac{5}{3} \lambda^{2}\left\langle\vec{\pi}^{2}(0)\right\rangle+m_{\pi}^{2} .
$$

One expects the exponential growth of the low-momentum modes for which the mass term is negative. This phenomenon is characterized as spinodal decomposition [13, 14].

b) At later stages the mass term becomes

$$
m_{e f f}^{2}(t)=k^{2}-\lambda^{2} v^{2}+\lambda^{2} \sigma^{2}(t)+\frac{5}{3} \lambda^{2}\left\langle\vec{\pi}^{2}(t)\right\rangle+m_{\pi}^{2}
$$

with a $\sigma(t)$ a quasi-periodic function. If the term $\sim\left\langle\vec{\pi}^{2}(t)\right\rangle$ is negligible the evolution equation becomes a Lamé or Mathieu equation (see Appendix B for a detailed discussion of this point and a related controversy). One expects a resonance band with exponential amplification of $f_{k}$. This phenomenon is characterized as parametric resonance. It has been studied in detail in relation to the reheating of the universe through inflaton decay [5, 6, 8, 15].

The analytical solution that we presented above, as well as the numerical analysis in the next section, demonstrate that there is no well-defined boundary separating periods in the time evolution during which one of the two mechanisms dominates. In general, there is a fast amplitude growth for the modes in a certain momentum zone (such as in equation (20) ). However, the phenomenon can be a complicated convolution of both mechanisms we discussed above. 


\section{NUMERICAL RESULTS AND DISCUSSION}

We solve equations (5) and (6) numerically using a fourth-order Runge-Kutta algorithm for the differential equations and an 11-point Newton-Gotes integrator to compute the momentum integral:

$$
\left\langle\vec{\pi}^{2}(t)\right\rangle=\int \frac{d^{3} k}{(2 \pi)^{3}} \vec{f}_{k}^{\star}(t) \vec{f}_{k}(t)=\int \frac{d k}{2 \pi^{2}} k^{2} \vec{f}_{k}^{\star}(t) \vec{f}_{k}(t) .
$$

We calculate the pion density in $3 D$ momentum space using $(8)$ and the total number of produced pions $N_{\text {tot }}$ using (9). Furthermore, it is convenient to define the projected $1 D$ density $\rho(k)$ through:

$$
N_{t o t}=\int_{0}^{\infty} d k \rho(k)
$$

The pion gas is initially a fireball at thermal equilibrium with temperature $T \simeq 140 \mathrm{MeV}$ and radius $r_{0} \simeq 10 \mathrm{fm}$. The quench is described by an instantaneous expansion of this fireball by a factor $\Lambda$. We consider here three different values of the final radius corresponding to small $\left(r_{f}=11 \mathrm{fm}(\Lambda=1.3)\right)$, intermediate $\left(r_{f}=12.6 \mathrm{fm}(\Lambda=2)\right)$ and large $\left(r_{f}=15 \mathrm{fm}(\Lambda=3.375)\right)$ expansion of the initial fireball. The effect of the quench is incorporated in the normalization of the pion mode functions $\vec{f}_{k}$ at $t=0$. Performing the numerical integration we obtain the results presented in Figs. 1-8, which are close to our analytical calculations described in section IV.

In Fig. 1 we depict the $\sigma$ evolution and the total pion number for expansion factor $\Lambda=3.375$. The radius of the expanded fireball in this case is $15 \mathrm{fm}$. During the first two oscillations of $\sigma(t)$ the number of produced pions $N_{t o t}$ increases fast, because of parametric resonance. This lasts about $6 \mathrm{fm}$ and subsequently $N_{\text {tot }}$ just fluctuates around a mean value $\simeq 830$. As our approach is semiclassical this solution describes the evolution of the system consistently only within the first stage $(t \lesssim 6 \mathrm{fm})$. During this time the pion production can be described through the energy transfer from the oscillating $\sigma$-field to the pionic field. In the following stages, our solution displays a periodic energy exchange between the two fields. A proper quantum treatment is necessary in order to describe the complete decay of the $\sigma$-field into free pions. However, the bulk of pions is produced during the first stage. For this reason we estimate the various observables (such as pion number and distribution) at a time $t \simeq 6 \mathrm{fm}$.

In Fig. 2 the evolution of the distribution of pions $\rho(k)$ for various times is shown. We observe a large enhancement of the spectrum at low momenta, and the formation of a zone with a peak at a specific $k$-value $\left(k_{\max }\right)$ and a width characteristic of the non-equilibrium amplification. Comparing with Fig. 1 we observe that the energy transfer to the pions is translated to a decrease of the $\sigma$-oscillation amplitude. In Fig. 2 we also observe a slight sinking of the spectrum at large momenta, which implies an additional energy transfer from hard to soft pion modes through the mode-mode coupling (the $\left\langle\vec{\pi}^{2}(t)\right\rangle$ term) in (6), effect that cannot be described within our approximations.

This behaviour can be described very well using the analytical results of section IV. For an expansion factor $\Lambda=3.375$ (and more generally for $\Lambda>3$ ) the initial values $f_{k i}(0)$ (according to (13)) are very small so that $\left\langle\vec{\pi}^{2}(0)\right\rangle \ll v^{2}$, justifying the conditions for parametric resonance: while $\sigma$ oscillates around its new minimum after the quench, it leads the pion mode functions in the momentum zone (20) to exponential increase. The numerical values for the limits of the momentum zone, whithin which the exponential amplification occurs, agree with a $4 \%$ accuracy with the analytical estimate of equation (20). This is explained by the fact that $\left\langle\vec{\pi}^{2}(t)\right\rangle$ is negligible initially in this case, so that the analytical solution gives a good approximation. As time passes, $\left\langle\vec{\pi}^{2}(t)\right\rangle$ increases, so that eventually the parametric resonance ends. Even though this effect cannot be described within the analytical approach, its influence on the location of the amplification zone is very small.

We also point out that the initial conditions for $\sigma(t)$ are determined by equation (12), so that a large $\Lambda$ corresponds to $\sigma(0)$ closer to $v$. This large initial value of $\sigma$ shifts the pion effective mass at $t=0$ to larger values, leading to a strong suppression of spinodal decomposition in favour of parametric resonance.

In Fig. 3 we plot $\sigma(t)$ and $N_{t o t}(t)$ for $\Lambda=1$.3. We observe that the pion production completes during the rolling down of the $\sigma$-field towards the new minimum. This process lasts for $\simeq 2 \mathrm{fm}$, and subsequently $N_{\text {tot }}$ oscillates in a manner similar to that in the case $\Lambda=3.375$. Observables are now considered for times $t \simeq 2 \mathrm{fm}$. For small expansion factors $(\Lambda<1.5)$ the dominant amplification mechanism is the spinodal decomposition: during the first rolling down of the $\sigma$-field towards its minimum, the effective squared pion mass (21) is negative and the pion modes with small momenta are amplified. Furthermore, because of the large value of $\left\langle\vec{\pi}^{2}(0)\right\rangle$, the requirements of parametric resonance are not fulfilled in the second stage when $\sigma(t)$ oscillates around the new minimum. As a result the amplification through parametric resonance is suppressed. We point out that the analytical solution of the previous section does not give a good quantitative approximation in this case, as the effect of the term $\sim\left\langle\vec{\pi}^{2}(t)\right\rangle$ cannot be neglected.

For completeness, we show in Fig. 4 the evolution of the pion distribution for $\Lambda=1.3$. Similarly to the previous case (Fig. 2) we observe an enhancement of the pion spectrum at low momenta. The lower limit of the ampilified zone is at $k=0$, while the upper limit is time dependent. The peak of the particle density per momentum mode is 


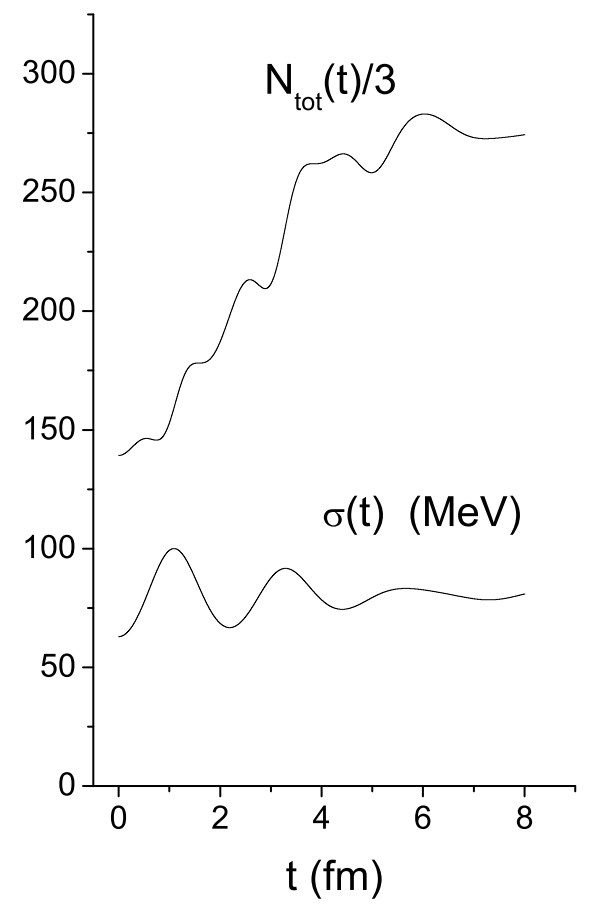

FIG. 1: The evolution of the $\sigma$-field as well as the total pion number $N_{t o t}(t)$ (scaled by a factor of 3 ) for initial radius 10 fm and final radius $15 \mathrm{fm}(\Lambda=3.375)$.

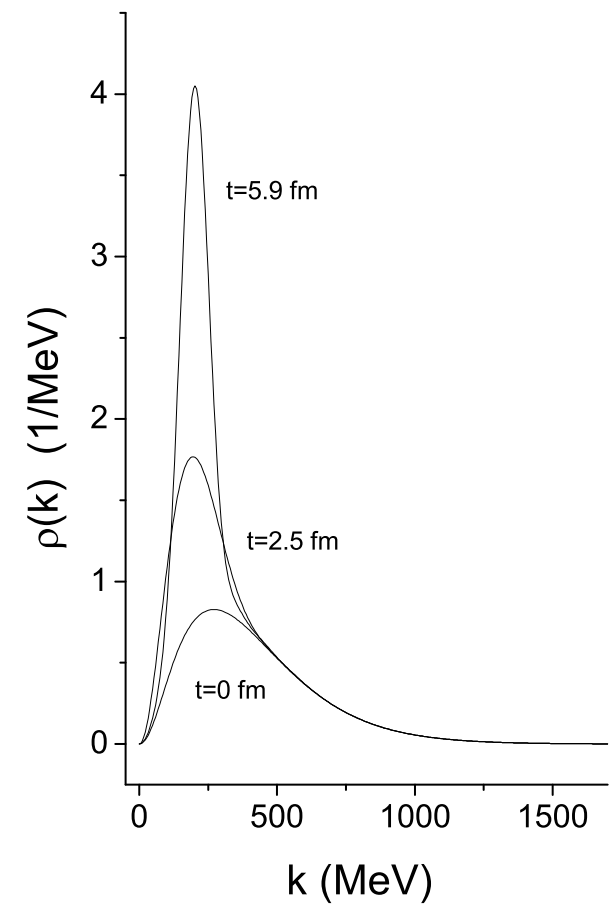

FIG. 2: The density $\rho(k)$ defined in equation (24) using $\Lambda=3.375$ at three successive times. 


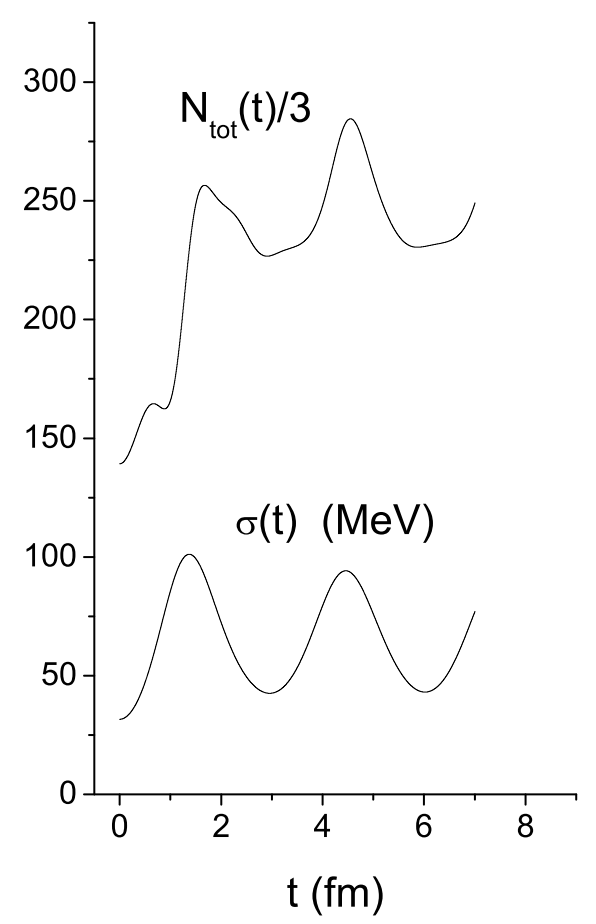

FIG. 3: The evolution of the $\sigma$-field as well as the total pion number $N_{t o t}(t)$ (scaled by a factor of 3 ) for expansion factor $\Lambda=1.3$.

at $k=0$. However this feature is modified at the level of one-particle momentum density (24), because of the phase space factor $4 \pi k^{2}$ that shifts the maximum to a non-zero momentum value.

Lastly, for intermediate expansion factors $(1.5<\Lambda<3)$ both amplification mechanisms are operative. During the first $\sigma$-rolling, $m_{\text {eff }}(t)$ is negative for low momenta and spinodal decomposition takes place. Subsequently, while $\sigma$ oscillates around its new minimum, $\left\langle\vec{\pi}^{2}(t)\right\rangle$ is not very large, allowing for parametric resonance to occur as well. This behaviour can be clearly seen in Fig. 5 : the pion production happens during both phases. At late times $\left\langle\vec{\pi}^{2}(t)\right\rangle$ grows significantly, parametric resonance ends, and pion production completes. The time when this happens is $\simeq 4 \mathrm{fm}$.

In this intermediate case both limits of the amplification zone are time dependent. As a result, the pion momentum spectrum changes with time. In fact, we get an oscillating zone in momentum space within which the amplification takes place. A simple explanation of this behaviour can be obtained if we observe that the addition of a constant term within the brackets in equation (18) shifts the amplification zone given by equation (20), to the left if the added term is positive, or to the right if it is negative. The addition of a fluctuating term $\left(\left\langle\vec{\pi}^{2}(t)\right\rangle\right)$ is expected to force the edges of the amplification zone to oscillate. It must be pointed out that, after the inclusion of the back-reaction term $\left\langle\vec{\pi}^{2}(t)\right\rangle$ in the equation for $\sigma$, the solution (16) is no longer valid. However, in this intermediate case the modification of the solution is not expected to be dramatic. The expected behaviour is observed in Fig. 6. The pion spectrum is enhanced and its maximum is shifted towards lower momenta as in the previous two cases. However, the location of the maximum of the spectrum and the limits of the amplification zone oscillate.

A common property of the momentum spectrum of the produced pions, for all the expansion factors we considered, is the appearance of an amplification zone in the region of low momenta. This results in the production of a relatively large number of soft pions during the evolution of the $\sigma-\pi$ system. As we have already mentioned, our semiclassical approach is not reliable for long times. We estimate the number of produced pions through the pion distribution at the beginning of the stage in which our solution predicts a periodic interchange of energy between the $\sigma$-field and pion sectors: $\simeq 6 \mathrm{fm}$ for $\Lambda=3.375, \simeq 4 \mathrm{fm}$ for $\Lambda=2$ and $\simeq 2 \mathrm{fm}$ for $\Lambda=1.3$. The effect of the amplification is quite strong, and persists even if we perform an averaging of the solution over times larger than the ones listed above. The energy density $\rho(k)$ is clearly distinguishable from that of a thermal system because of the presence of a narrow peak at low momenta.

The formation of the momentum amplification zone reflects a clustering of the pion momenta in the low $k$-region. The dynamical character of this cluster, which leads to the presence of a peak at a definite momentum value, accompanied by a width of typical size, in the projected one-particle density, is crucial. This characteristic form can 


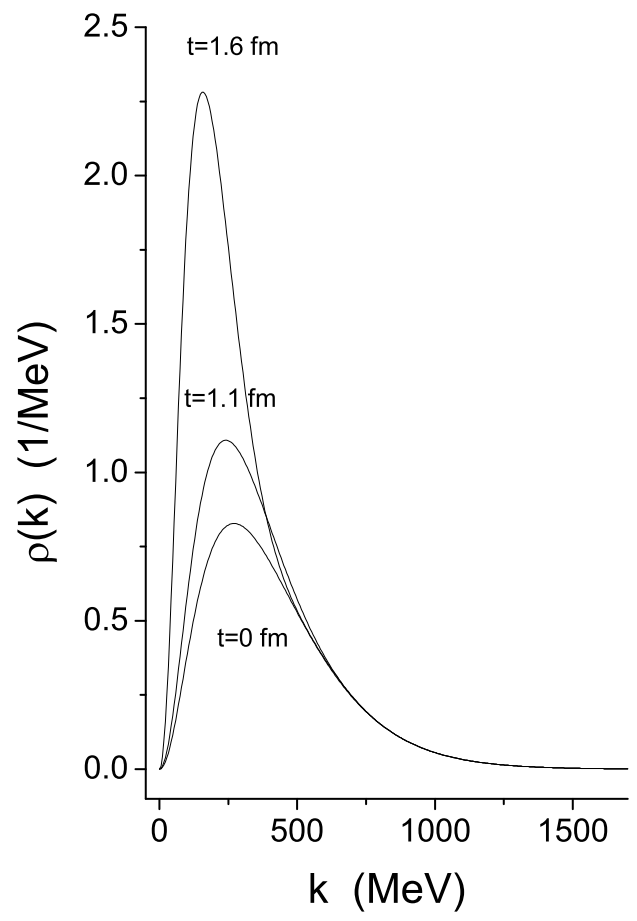

FIG. 4: The density $\rho(k)$ using $\Lambda=1.3$ at three successive times.

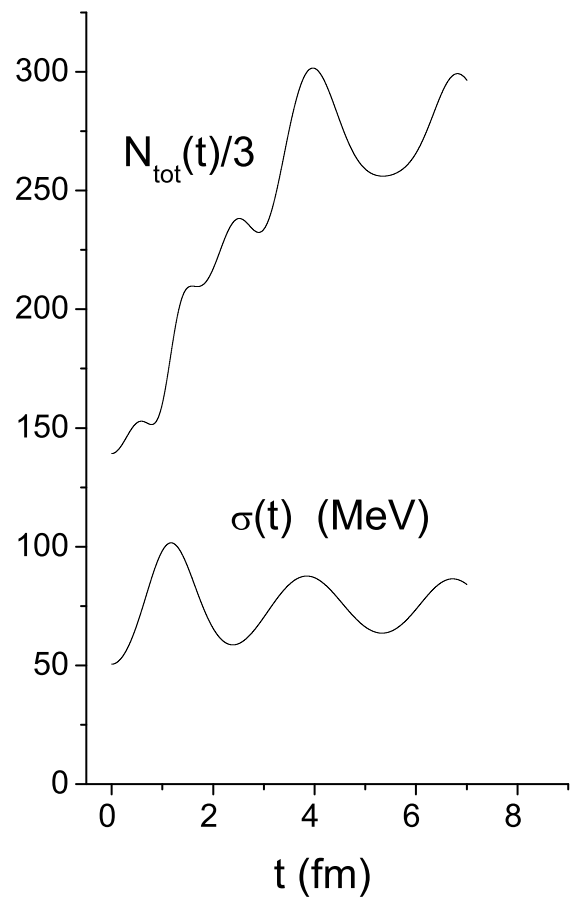

FIG. 5: The function $\sigma(t)$ as well as the total pion number $N_{t o t}(t)$ (scaled by a factor of 3) for an expansion factor $\Lambda=2$. 


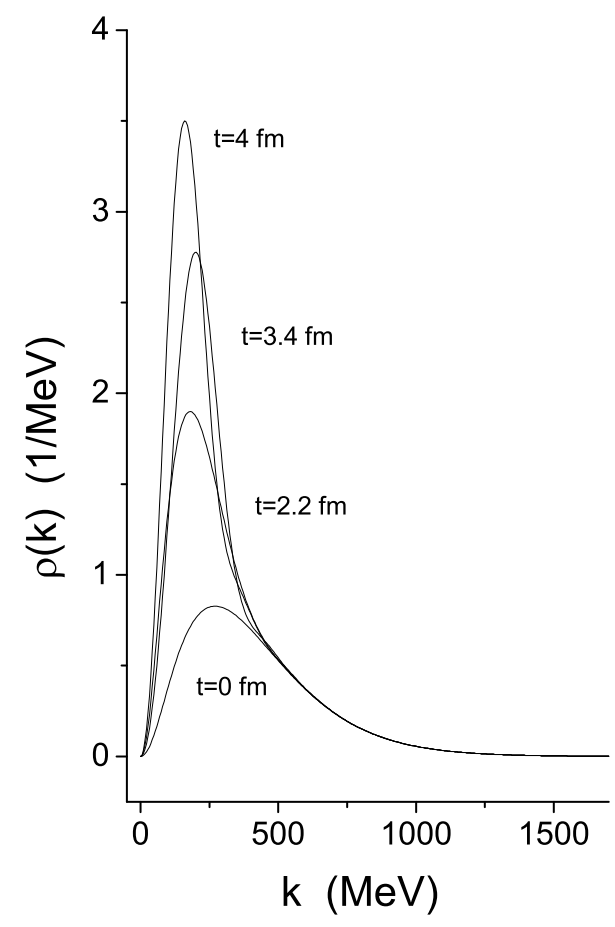

FIG. 6: The density $\rho(k)$ for the case of intermediate expansion $(\Lambda=2)$ at four successive times.

be directly observed in an inclusive analysis of the pion momenta produced for example in $A+A$ ultrarelativistic collisions. The geometry of this cluster is expected to be rather simple: no substructures at smaller scales are present. It is worth comparing the situation here with the corresponding formation of particle clusters in a critical system. In the latter case the clusters have statistical character. Therefore, at the level of one-particle inclusive density in momentum space no peaks (beyond the kinematic ones) appear. The geometry of these critical clusters is that of a random fractal: self-similar structures at different scales occur, leading to a characteristic power-law dependence of the factorial moments on the resolution scale $M$ [17].

In order to explore the resonant pion production at a phenomenological level, we have generated, through a MonteCarlo simulation of equations (92),(24), data sets consisting of a large number of events. For each value of the expansion factor $\Lambda$ that we considered, we produce 1000 events. We then calculated the corresponding second factorial moment in transverse momentum space as a function of the resolution scale $M[18]$. Our results are given in Fig. 7. We observe a conventional behaviour of the moments (saturation at high resolution scales) reflecting the absence of self-similar structure at different scales in the formed pionic clusters, for all expansion factors.

One could also consider the inclusive density of the transverse momentum for each set of 1000 events. We have calculated this distribution for the case $\Lambda=3.375$. The result is displayed in Fig. 8, where we clearly observe the effect of the amplification zone. In the same plot we show the experimentally observed transverse momentum distribution of the charged pions produced in $\mathrm{Pb}+\mathrm{Pb}$ collisions at $158 \mathrm{GeV} / \mathrm{n}$ (NA49-SPS) 19]. The experimental results are very close to a thermal system in transverse momentum space. The large deviation of the transverse momentum distribution of the pions produced through the amplification mechanisms described in the present work, from the corresponding distribution of a conventional thermal system, is an experimentally accessible signal for the appearance of non-equlibrium phenomena, associated with chiral phase transition, in the pion production during a heavy-ion collision.

\section{CONCLUSIONS}

In this work we studied non-equlibrium phenomena related to the chiral QCD phase transition. We found that, within the scenario of quench, the dynamics of the system may amplify pion modes in a certain momentum zone while the $\sigma$-field moves towards and around the (chirally broken) vacuum. These phenomena produce a significant 


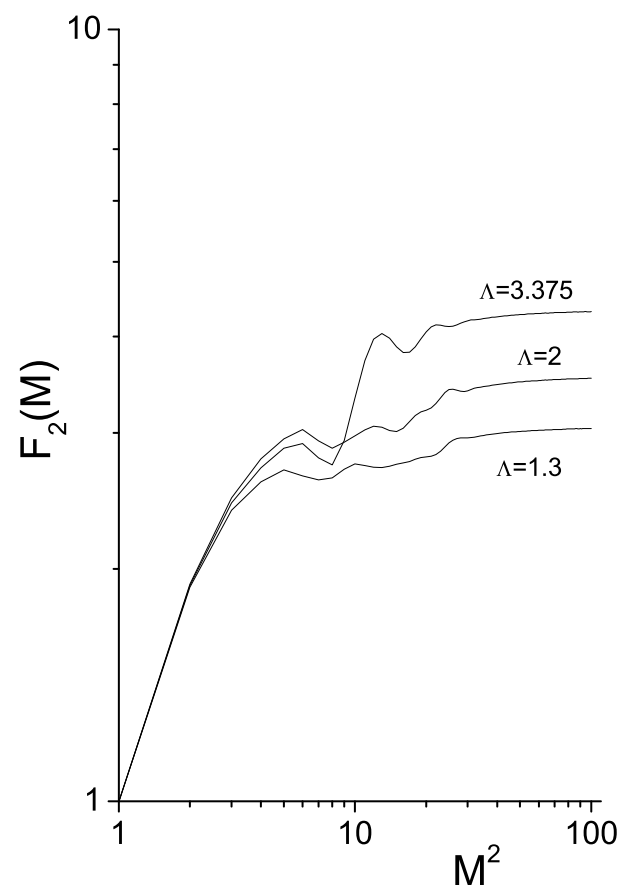

FIG. 7: The second factorial moment for the three different cases of large $(\Lambda=3.375)$, intermediate $(\Lambda=2)$ and small $(\Lambda=1.3)$ expansion. For each case we have analysed 1000 Monte-Carlo events generated according to equations (9), 24).

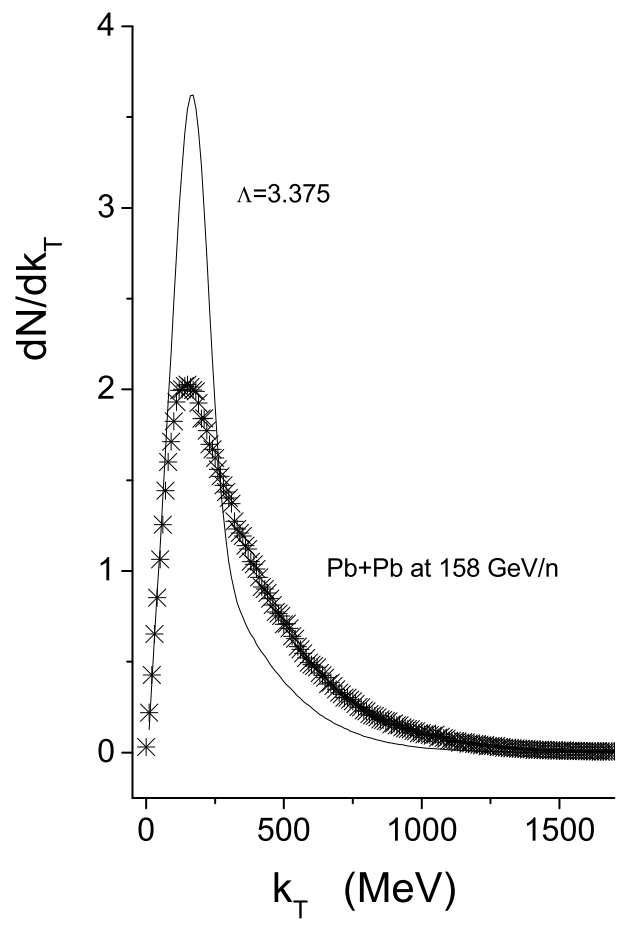

FIG. 8: The transverse momentum distribution of the finally produced pions in the case of large expansion $(\Lambda=3.375)$. For comparison the $k_{T}$-distribution for a system at thermal equilibrium $(P b+P b$ at $158 \mathrm{GeV} / \mathrm{n})$ measured at the NA49 experiment (CERN-SPS) is also displayed. Both distributions are normalized to the same total pion multiplicity. 
enhancement of the pion spectrum at low momenta. In our approach we took account of two main features of the physical system: the explicit symmetry breaking through the non-zero pion mass, and the local thermal equilibrium for the initial pion gas. In this sense our study is close to the real world. As a consequence, the pion production in our model is not as pronounced as in other models [6, 9, 10]. However, the momentum distribution of the produced pions possesses fingerprints of the zone structure of the amplification regions in momentum space: a cluster of pions with low momenta is formed. Although visible at the level of single particle density, this cluster does not have substructures at different scales. It is, therefore, distinguishable from particle clusters created by different mechanisms (equilibrium phase transition). Furthermore, the transverse momentum spectrum acquires a specific non-thermal peak that could be a clear signature of these non-equilibrium phenomena in a collision experiment with relativistic heavy ions.

It would be interesting to examine the modifications in the scenario we studied, if the finite time neccessary for the quench is taken into account. Additionally, one should also consider the consequences of the cylindrical form of the expanding fireball for the observed pion spectrum. Such a geometry is closer to the conditions occuring in $A+A$ experiments at very high energies. Another important aspect is the case when the initial state is critical, i.e. large self-similar fluctuations of the $\sigma$-field are present. In this case, the interesting problem is to explore the evolution of the critical state towards the freeze-out phase. The study of these additional issues is left for future investigations.

\section{Appendix A}

We present here the derivation of some of the results used in the main text. Equation (18) with $\varepsilon=0$ has the form

$$
f_{k i}^{\prime \prime}(\tau)+\left[\frac{k^{2}}{\lambda^{2} v^{2}}+\eta^{2}(\tau)-1\right] f_{k i}(\tau)=0
$$

with

$$
\begin{gathered}
\eta(\tau)=\frac{\eta(0)}{\operatorname{dn}\left(\tau \sqrt{1-\frac{n^{2}(0)}{2}}, q\right)}, \\
q=\sqrt{\frac{1-\eta^{2}(0)}{1-\frac{\eta^{2}(0)}{2}}} .
\end{gathered}
$$

Here $\eta(\tau)$ is a doubly periodic function with periods $2 \omega=2 K(q) / \sqrt{1-\frac{\eta^{2}(0)}{2}}\left(\right.$ real) and $4 \omega^{\prime}=4 i K^{\prime}(q) / \sqrt{1-\frac{\eta^{2}(0)}{2}}$ (complex), where $K(q)$ is the complete elliptic integral of first kind and $K^{\prime}(q)$ is its complementary.

Equation (25) is the Lamé equation, for a particular value of the coefficients that make it solvable in terms of Jacobi functions. It is convenient to express the Jacobi function $\mathrm{dn}$ in terms of the Weierstrass $\mathcal{P}$ function through 12 ]

$$
\frac{1}{\operatorname{dn}^{2}(u, q)}=\frac{1-\frac{1}{\operatorname{sn}^{2}\left(u+K(q)+i K^{\prime}(q)\right)}}{1-q^{2}}=\frac{1}{e_{1}-e_{2}}\left[e_{1}-\mathcal{P}\left(\frac{u+K(q)+i K^{\prime}(q)}{\sqrt{e_{1}-e_{3}}}\right)\right]
$$

where $q=\sqrt{\frac{e_{2}-e_{3}}{e_{1}-e_{3}}}$. The parameters $e_{1}, e_{2}, e_{3}$ are the roots of a cubic equation whose coefficients depend on the two periods of the Weierstrass $\mathcal{P}$ function [12]. They obey the relation $e_{1}+e_{2}+e_{3}=0$. Without loss of generality we choose $e_{1}-e_{3}=1-\frac{\eta^{2}(0)}{2}$. Thus we obtain

$$
\begin{gathered}
3 e_{1}=1 \\
3 e_{2}=1-\frac{3}{2} \eta^{2}(0) \\
3 e_{3}=\frac{3}{2} \eta^{2}(0)-2 .
\end{gathered}
$$

Inserting (28) into (25) and using the definitions of the periods $\omega, \omega^{\prime}$, we find

$$
f_{k i}^{\prime \prime}(\tau)+\left[\frac{k^{2}}{\lambda^{2} v^{2}}-\frac{1}{3}-2 \mathcal{P}\left(\tau+\omega+\omega^{\prime}\right)\right] f_{k i}(\tau)=0 .
$$

The solutions of (30) are of the form [1]

$$
f_{k i}(\tau)=\frac{\sigma\left(\tau+\omega+\omega^{\prime}+\mathrm{w}(k)\right)}{\sigma\left(\tau+\omega+\omega^{\prime}\right)} e^{-\tau \zeta(\mathrm{w}(\mathrm{k}))},
$$


where $\sigma(x)$ and $\zeta(x)$ are the known Weierstrass functions defined as

$$
\zeta(u)=\frac{1}{u}-\int_{0}^{u}\left(\mathcal{P}(z)-\frac{1}{z^{2}}\right) d z
$$

and

$$
\sigma(u)=u \exp \left\{\int_{0}^{u}\left(\zeta(z)-\frac{1}{z}\right) d z\right\}
$$

and the complex number $\mathrm{w}(k)$ is defined through the transcendental equation

$$
\mathcal{P}(\mathrm{w})=-\frac{k^{2}}{\lambda^{2} v^{2}}+\frac{1}{3}
$$

Because of the quasi-periodicity property of the $\sigma$-function [12]

$$
\sigma(x+2 \omega)=-\sigma(x) e^{2(x+\omega) \zeta(\omega)},
$$

we obtain from 31

$$
f_{k i}(\tau+2 \omega)=f_{k i}(\tau) e^{2[\mathrm{w}(k) \zeta(\omega)-\omega \zeta(\mathrm{w}(k))]} .
$$

As a result, for $\mathrm{w}(k)$ for which the exponent

$$
\mu(\mathrm{w}(k))=2[\mathrm{w}(k) \zeta(\omega)-\omega \zeta(\mathrm{w}(k))]
$$

becomes real, we have an exponential amplification of $f_{k i}(\tau)$. For values of $\mathrm{w}(k)$ which lead to imaginary $\mu(\mathrm{w}(k))$ we obtain oscillatory behaviour.

To determine the corresponding $\mu(\mathrm{w}(k))$ intervals one has to consider the equation (34) that maps the real $k^{2} /\left(\lambda^{2} v^{2}\right)$ axis onto the sides of the fundamental square in the w plane [6, 12].

- If $-\infty \leq \frac{k^{2}}{\lambda^{2} v^{2}} \leq 0$, then $\mathrm{w}(k)=\beta \in R$ with $0 \leq \beta \leq \omega$.

- If $0 \leq \frac{k^{2}}{\lambda^{2} v^{2}} \leq \frac{\eta^{2}(0)}{2}$, then $\mathrm{w}(k)=\omega+i \alpha(\alpha \in R)$ with $0 \leq \alpha \leq-i \omega^{\prime}$.

- If $\frac{\eta^{2}(0)}{2} \leq \frac{k^{2}}{\lambda^{2} v^{2}} \leq 1-\frac{\eta^{2}(0)}{2}$, then $\mathrm{w}(k)=\omega^{\prime}+i \beta$ with $\omega \geq \beta \geq 0$.

- If $1-\frac{\eta^{2}(0)}{2} \leq \frac{k^{2}}{\lambda^{2} v^{2}} \leq \infty$, then $\mathrm{w}(k)=i \alpha$ with $-i \omega^{\prime} \geq \alpha \geq 0$.

The appearance of $\eta(0)$ in these four intervals results from the dependence of the roots $e_{1}, e_{2}, e_{3}$ in (29) on $\eta(0)$. Using the properties of the $\zeta$-function 12 we can easily derive that $\mu(\mathrm{w}(k))$ has a non-zero real positive part for the cases (A) and (C), while it is purely imaginary for (B) and (D).

\section{Appendix B}

We derive the Mathieu equation starting from the Lamé equation. We use the expansion 12$]$

$$
\frac{1}{\operatorname{dn}(u, q)}=\frac{\pi}{2 q^{\prime} K(q)}\left[1+4 \sum_{n=1}^{\infty}(-1)^{n} \frac{Q^{n}}{1+Q^{2 n}} \cos \frac{n \pi u}{K(q)}\right]
$$

where

$$
Q \equiv e^{-\frac{\pi K^{\prime}(q)}{K(q)}}
$$

is the elliptic nome. This expansion holds for $\operatorname{Im}\left(\frac{\pi u}{2 K(q)}\right)<\frac{1}{2} \pi \operatorname{Im}\left(\frac{i K^{\prime}(q)}{K(q)}\right)[20] . Q$ can be expanded as $[12]$

$$
Q=e^{-\frac{\pi K^{\prime}(q)}{K(q)}}=\lambda+2 \lambda^{5}+15 \lambda^{9}+150 \lambda^{13}+\cdots
$$


where

$$
\lambda=\frac{1}{2} \frac{1-\sqrt{q^{\prime}}}{1+\sqrt{q^{\prime}}}=\frac{1}{2} \frac{1-\left(1-q^{2}\right)^{1 / 4}}{1+\left(1-q^{2}\right)^{1 / 4}} .
$$

$K(q)$ can be also expanded as

$$
K(q)=\frac{\pi}{2} \sum_{n=1}^{\infty}\left(\frac{(2 n-1) ! !}{2^{n} n !}\right)^{2} q^{2 n}
$$

Keeping only the first terms in the expansions we obtain

$$
\eta^{2}(\tau)=\frac{\eta^{2}(0)}{\operatorname{dn}^{2}\left(\tau \sqrt{1-\frac{n^{2}(0)}{2}}, q\right)}=\frac{\eta^{2}(0) \pi}{2\left(1-\frac{1}{3} q^{2}-\frac{7}{64} q^{4}\right)}\left[1-8\left(\lambda+2 \lambda^{5}\right) \cos \left(\frac{2 \tau \sqrt{1-\frac{n^{2}(0)}{2}}}{1+\frac{1}{4} q^{2}+\frac{9}{64} q^{4}}\right)\right]
$$

with $q=\sqrt{\frac{1-\eta^{2}(0)}{1-\frac{\eta^{2}(0)}{2}}}$. Substituting this in the Lamé equation (25) we find

$$
\left[\frac{d^{2}}{d z^{2}}+A-2 B \cos 2 z\right] f_{k i}(z)=0
$$

with

$$
\begin{aligned}
A & =\frac{k^{2}}{\lambda^{2} v^{2}}+2 \varepsilon-1+\frac{\eta^{2}(0) \pi}{2\left(1-\frac{1}{3} q^{2}-\frac{7}{64} q^{4}\right)} \\
B & =2 \frac{\eta^{2}(0) \pi}{\left(1-\frac{1}{3} q^{2}-\frac{7}{64} q^{4}\right)}\left(\lambda+2 \lambda^{5}\right) \\
z & =\frac{\tau \sqrt{1-\frac{n^{2}(0)}{2}}}{1+\frac{1}{4} q^{2}+\frac{9}{64} q^{4}} .
\end{aligned}
$$

Equation (41) is the Mathieu equation. It is a good approximation of the Lamé equation for $Q \ll 1$, i.e. for $q \ll 1$, since the elliptic functions approach the usual trigonometric ones for $q \rightarrow 0$. However, in the case $Q \sim 1$, i.e for $q \sim 1$, all higher-order terms in (37) must be taken into account. The simple approximation that leads to the Mathieu equation is not valid. There is some controversy in the literature [16] on whether the Mathieu equation gives a reliable approximation in the studies of parametric resonance during inflaton decay.

\section{Acknowledgments}

This work was partly supported by the Research Committee of the University of Athens and the Hellenic State Scholarships Foundation (IKY).

[1] K. Rajagopal and F. Wilczek, arXiv:hep-ph/0011333

[2] M. Gell-Mann and M. Levy, Nuovo Cim. 16 (1960) 705.

[3] K. Rajagopal and F. Wilczek, Nucl. Phys. B 399 (1993) 395 arXiv:hep-ph/9210253.

[4] J. T. Lenaghan and D. H. Rischke, J. Phys. G 26 (2000) 431 arXiv:nucl-th/9901049.

[5] L. Kofman, A. D. Linde and A. A. Starobinsky, Phys. Rev. Lett. 73 (1994) 3195 arXiv:hep-th/9405187.

[6] D. Boyanovsky, H. J. de Vega, R. Holman and J. F. Salgado, Phys. Rev. D 54 (1996) 7570 arXiv:hep-ph/9608205.

[7] J. Berges, D. U. Jungnickel and C. Wetterich, Phys. Rev. D 59 (1999) 034010 arXiv:hep-ph/9705474.

[8] D. Boyanovsky, H. J. de Vega and R. Holman, arXiv:hep-ph/9701304

[9] D. I. Kaiser, Phys. Rev. D 59 (1999) 117901 arXiv:hep-ph/9801307.

[10] A. Dumitru and O. Scavenius, Phys. Rev. D 62 (2000) 076004 arXiv:hep-ph/0003134.

[11] E. L. Ince, Ordinary Differential Equations, Dover Publications, New York (1926). 
[12] I. S. Gradshteyn and I. M. Ryzhik, Tables of Integrals, Series and Products, Academic Press, Orlando (1965).

[13] G. Brown, P. A. Rikvold, M. Sutton and M. Grant, Phys. Rev. E 60 (1999) 5151 arXiv:cond-mat/99905343].

[14] D. Boyanovsky, D. s. Lee and A. Singh, Phys. Rev. D 48 (1993) 800 arXiv:hep-th/9212083.

[15] Y. Shtanov, J. Traschen and R. H. Brandenberger, Phys. Rev. D 51 (1995) 5438 arXiv:hep-ph/9407247.

[16] L. Kofman, A. D. Linde and A. A. Starobinsky, arXiv:hep-ph/9608341,

D. Boyanovsky, H. J. de Vega and R. Holman, arXiv:hep-ph/9609366

[17] N. G. Antoniou, Y. F. Contoyiannis and F. K. Diakonos, Phys. Rev. E 62 (2000) 3125 arXiv:hep-ph/0008047; N. G. Antoniou, Y. F. Contoyiannis, F. K. Diakonos, A. I. Karanikas and C. N. Ktorides, Nucl. Phys. A 693 (2001) 799 arXiv:hep-ph/0012164.

[18] A. Bialas and R. Peschanski, Nucl. Phys. B 308 (1988) 857.

[19] T. Alber et al. [NA49 Collaboration], Prepared for 25th International Symposium on Multiparticle Dynamics, Stara Lesna, Slovakia, 12-16 Sep 1995.

[20] This is always satisfied in our model, since $q=\sqrt{\frac{1-\eta^{2}(0)}{1-\frac{\eta^{2}(0)}{2}}}$ with $0<\eta(0)<1$, so that $q, K(q), K^{\prime}(q)$ are always real. 